\title{
Inverse photoemission in strongly correlated electron systems
}

\author{
R. Eder ${ }^{1}$ and Y. Ohta ${ }^{2}$ \\ ${ }^{1}$ Department of Applied and Solid State Physics, University of Groningen, 9747AG Groningen, The Netherlands \\ ${ }^{2}$ Department of Physics, Chiba University, Chiba 263, Japan
}

\begin{abstract}
Based on exact results for small clusters of $t-J$ model we point out the existence of several distinct channels in the inverse photoemission (IPES) spectrum. Hole-like quasiparticles can either be annihilated completely, or leave behind a variable number of spin excitations, which formed the dressing cloud of the annihilated hole. In the physical parameter regime the latter processes carry the bulk of IPES weight and although the Fermi surface takes the form of hole pockets, the distribution of spectal weight including the 'spin excitation bands' is reminiscent of free electrons.
\end{abstract}

74.20.-Z, 75.10.Jm, 75.50.Ee

A frequently used concept in many-body physics is the 'dressing' of a particle by an excitation cloud. Sudden annihilation of the particle then may leave behind its dressing cloud, and these 'released' excitations may carry excess momentum and energy. Such 'shake-off' processes represent a channel for particle annihilation of pure many-body origin, and one may expect that their relative importance depends on the 'degree of dressing', as measured by the quasiparticle weight $Z$. To see this, let us consider the change of the single particle spectral function upon removing an (infinitesimal) fraction $\epsilon$ of electrons/spin direction. In a noninteracting system thereby the topmost $N \epsilon$ poles of the photoemission (PES) spectrum cross to the inverse photoemission (IPES) spectrum ( $N$ denotes the system size). In a Fermi liquid the same will happen; depending on the magnitude of $Z$, however, the corresponding decrease of the integrated PES weight of $N \epsilon \cdot Z$ may be substantially less than the change of $N \epsilon$ required to maintain the correct electron count (the integrated PES weight equals the number of electrons). Spectral weight corresponding to $N \epsilon(1-Z)$ electrons therefore must shift from PES to IPES at momenta and energies off the Fermi surface, and a natural mechanism to accomplish this would be the 'shake-off processes' adressed above. Values of $Z \approx 0.2-0.5$, as found in the $t-J$ and Hubbard model near half-filling [1], thus suggest that in this case in fact the bulk of IPES weight is carried by shake-off processes. In this manuscript we present diagonalization results which substantiate this general argument. The emerging picture of the single particle spectral function and Fermi surface not only resolves the apparent contradiction between mounting evidence [2,3] for a hole pocket-like Fermi surface in these models on one hand and the roughly free electron-like weight distribution in their spectral function [4] on the other; it is also reasonably consistent with experiments on cuprate superconductors.

For the standard $t-J$ model [1] we study the electron addition spectrum

$$
A_{+}(\boldsymbol{k}, \omega)=\sum_{\nu}\left|\left\langle\nu_{n}\left|\hat{c}_{\boldsymbol{k}, \uparrow}^{\dagger}\right| 0_{n+1}\right\rangle\right|^{2} \delta\left(\omega-\omega_{\nu}^{n}\right)
$$

where $\left|\nu_{n}\right\rangle$ denotes the $\nu^{t h}$ eigenstate with $n$ holes (in particular $\nu=0$ implies the ground state (GS)) and $\omega_{\nu}^{n}$ its excitation energy relative to the $n$-hole GS. The operator $\hat{c}_{\boldsymbol{k}, \sigma}^{\dagger}$ is the Fourier transform of $\hat{c}_{i, \sigma}^{\dagger}=c_{i, \sigma}^{\dagger}\left(1-n_{i,-\sigma}\right)$. where $c_{i, \sigma}^{\dagger}$ denotes the Fermion creation operator. For small clusters $A_{+}(\boldsymbol{k}, \omega)$ can be evaluated exactly by the Lanczos algorithm, the results presented below have been obtained in the standard 18-site cluster [1], results for the 16 site cluster are consistent with these.

As a first step we consider the dependence of $A_{+}(\boldsymbol{k}, \omega)$ on $J / t$ and hole number $n_{h}$. A trivial dependence on $n_{h}$ is due the requirement that the $\boldsymbol{k}$ and $\omega$ integrated IPES weight equals $n_{h}$. Figure 1 therefore shows the IPES spectra divided by $n_{h}$ for the 1 and 2 hole ground states and various $J / t$. The single hole GS is 8 -fold degenerate, because it has finite momentum, $\boldsymbol{k}_{0}=( \pm 2 \pi / 3,0),(0, \pm 2 \pi / 3)$, and $z$-spin $S_{z}= \pm 1 / 2$; the single hole spectra of Figure 1 have been obtained by averaging over these 8 degenerate states. When energies are measured in units of $J$, it is immediately obvious that apart from an overall slight shift to lower energies in the 2-hole case (which may reflect the 'softening' of the spin excitations due to increased hole doping) the two spectra are remarkably similar: they show 'features' at comparable energies, with comparable spectral weight and with an anlogous dependence of the weight on $\mathrm{J} / \mathrm{t}$ (analogous features can also be seen in the IPES spectra of the 16-site cluster). Up to the 'softening' of peak energies, the IPES spectrum thus scales with $n_{h}$ over its entire width, in strong contrast e.g. to noninteracting electrons, where the parts far from the Fermi energy remain unaffected by a change of electron density. We thus see the same remarkable continuity with hole doping as established previously for the PES spectrum [2] and the dynamical charge correlation function [5].

We proceed to a detailed examination of the single hole case. More precisely, we study the addition of an $\uparrow$ spin electron to the single-hole GS with momentum $(-2 \pi / 3,0)$ and $z$-spin $-1 / 2$; the final states are eigenstates of the undoped antiferromagnet, i.e. spin excited states, with $z$-spin $S_{z}=0$ and total spin $S_{t o t}=0,1$. 
We define the spin excitation operators $S_{1, j}=S_{j}^{z}$ and $S_{2, j}=\boldsymbol{S}_{j} \cdot \boldsymbol{S}_{j+\hat{x}}+\boldsymbol{S}_{j} \cdot \boldsymbol{S}_{j+\hat{y}}$ (where e.g. $j+\hat{x}$ denotes the nearest neighbor of site $j$ in $x$-direction) and their Fourier transforms $S_{\lambda, \boldsymbol{q}}$. Then, the following off-diagonal Green's functions describe the interference between IPES in the $n+1$ hole GS and spin excitation of the $n$ hole GS:

$$
B_{\lambda}(\boldsymbol{k}, \omega)=\sum_{\nu, \boldsymbol{q} \neq 0} \frac{\left\langle 0_{n}\left|S_{\lambda, \boldsymbol{q}}^{\dagger}\right| \nu_{n}\right\rangle\left\langle\nu_{n}\left|c_{\boldsymbol{k}, \sigma}^{\dagger}\right| 0_{n+1}\right\rangle}{\left\langle 0_{n}\left|S_{\lambda, \boldsymbol{q}}^{\dagger} S_{\lambda, \boldsymbol{q}}\right| 0_{n}\right\rangle^{1 / 2}} \delta\left(\omega-\omega_{\nu}^{n}\right) .
$$

We have normalized the state $S_{\lambda, \boldsymbol{q}}\left|0_{n}\right\rangle$ to unity in order to suppress a possibly strong $\boldsymbol{q}$ dependence of its norm; in evaluating $B_{\lambda}(\boldsymbol{k}, \omega)$ we moreover readjusted for each $\boldsymbol{k}$ the (arbitrary) relative phase between the ground states $\left|0_{0}\right\rangle$ and $\left|0_{1}\right\rangle$ such that the frequency integral of $B_{\lambda}(\boldsymbol{k}, \omega)$ is real and positive. The imaginary part then vanishes identically. Figure 2 a shows $A_{+}(\boldsymbol{k}, \omega)$ and $\left|\Re B_{\lambda}(\boldsymbol{k}, \omega)\right|$. In the IPES spectrum $A_{+}(\boldsymbol{k}, \omega)$ there is precisely one peak with excitation energy $\omega_{\nu}=0$ at the position of the 'hole momentum', $(2 \pi / 3,0)$. The corresponding final state is the GS of the undoped system, so that this peak obviously describes the inverse process of hole creation in the half-filled GS. In a single particle picture, it would be the only peak expected. In the actual IPEs spectrum, however, the bulk of weight is carried by peaks with finite excitation energy and comparison with $B_{\lambda}(\boldsymbol{k}, \sigma)$ establishes a hierarchy of increasing spin excitation: the lowest group of the finite energy peaks corresponds to a single spin wave left behind in the cluster, the next highest group to a 'bimagnon' and most probably even higher peaks correspond to an even stronger spin excitation left behind by the annihilated hole. As regards the Fermi surface, it is clear that only the lowest state in this hierarchy, corresponding to "complete annihilation' of the quasiparticle should be considered. The IPES spectrum thus consists of the 'hole pocket' at $(2 \pi / 3,0)$ [3] plus the 'shake off bands' at higher energies. The latter are present throughout the Brillouin zone, however with a strongly reduced spectral weight near $\Gamma$; this is to be expected from the kinetic energy sum rule $E_{k i n}=-\sum_{\boldsymbol{k}} \epsilon_{\boldsymbol{k}} W_{\boldsymbol{k}}^{I P E S}$, where $\epsilon_{\boldsymbol{k}}$ denotes the noninteracting kinetic energy and $W_{\boldsymbol{k}}^{I P E S}$ the integrated IPES weight for momentum $\boldsymbol{k}$ : large (small) IPES weight near the zone boundary (zone center) optimizes the kinetic energy.

We proceed to the two-hole case. The GS in this case is a spin singlet and has $d_{x^{2}-y^{2}}$ symmetry; introducing $\boldsymbol{k}_{1}=(2 \pi / 3,0)$ and $\boldsymbol{k}_{2}=(0,2 \pi / 3)$, the simplest twoparticle state with these quantum numbers to be expected within a rigid-band picture would be:

$$
\begin{aligned}
\left|\Psi_{0}\right\rangle=\frac{1}{2}\left[a_{k_{1}, \uparrow}^{\dagger} a_{-k_{1}, \downarrow}^{\dagger}-a_{k_{1}, \downarrow}^{\dagger} a_{-k_{1}, \uparrow}^{\dagger}\right. & \\
& \left.-a_{k_{2}, \uparrow}^{\dagger} a_{-k_{2}, \downarrow}^{\dagger}+a_{k_{2}, \downarrow}^{\dagger} a_{-k_{2}, \uparrow}^{\dagger}\right]|v a c\rangle .
\end{aligned}
$$

For this state, the 'quasiparticle occupation' of the 4 equivalent momenta $\pm \boldsymbol{k}_{1}, \pm \boldsymbol{k}_{2}$ is the same for each spin direction. Thus if we make the simplest assumption possible, namely that the annihilation of one hole is not at all influenced by the presence of the second one, the twohole IPES spectrum should simply be the average of the single hole IPES spectra for the different momenta and spin directions, as is indeed the case. Figure $2 \mathrm{~b}$ shows the 'interference spectrum' $\left|\Re B_{\lambda}(\boldsymbol{k}, \omega)\right|$ for $n=1$ (the latter spectrum being averaged over the 4 degenerate single hole ground states with spin $1 / 2$ ). Unlike the $n=0$ case, the IPES peaks now show overlap with both types of spin excitations - this is to be expected, because the extra spin defects generated by the operators $S_{\lambda, \boldsymbol{q}}$ in the single hole GS may also be 'absorbed' into the dressing cloud of the hole. For example, addition of a spin excitation with momentum $(2 \pi / 3,2 \pi / 3)$ to the single hole GS with momentum $(-2 \pi / 3,0)$ with a certain probability may simply give the degenerate GS with momentum $(0,2 \pi / 3)$. Apart from this slight complication, however, the structure of $B_{\lambda}(\boldsymbol{k}, \omega)$ is quite similar to the single hole case: the single spin wave operator $S_{1}$ has strongest overlap with the dominant peak at the bottom of the respective IPES spectrum, whereas the smaller IPES peaks at higher energies rather correspond to the 'bimagnon': obviously there is the same hierarchy of increasing spin excitation as in the single hole case (as is to be expected from the simple scaling of the IPES spectrum with $n_{h}$, compare Figure 1.

Taken together, the numerical data suggest the existence of different 'channels' in the IPES spectrum: in addition to a 'conventional channel' at the lowest excitation energies, where a hole-like quasiparticle is 'annihilated completely', there exist higher energy final states where the annihilation process of the quasiparticle leaves behind a variable number of spin excitations, which formed the dressing cloud of the annihilated hole. The high degree of continuity of the IPES spectrum with doping moreover suggests that the spin-bag like quasiparticles for the half-filled case persist as well-defined entities also in the two-hole case (i.e. at nominal hole concentrations $\delta \approx 12 \%$ ). Assuming that these different channels remain 'disconnected' in the infinite system, i.e. that they do not merge to form a single 'band', the Fermi surface therefore should take the form of hole pockets, consistent with increasing numerical evidence [3]. The emerging picture of the low energy weight distribution and Fermiology in the full single particle spectral function then is summarized in Figure 3a (thereby the incoherent continua deep below the Fermi energy are omitted). To begin with, the Fermi surface is a hole pocket [3] generated by shifting the chemical potential into the more or less rigid nextnearest neighbor hopping band for hole motion in the undoped antiferromagnet [2]. Thereby the PES spectral weight of the quasiparticle band in the outer parts of the Brillouin zone is small [2, 6]; this 'shadow band' [7] effect is readily understood [2] by the corollary of the kinetic 
energy sum-rule, $E_{k i n}=\sum_{\boldsymbol{k}} \epsilon_{\boldsymbol{k}} W_{\boldsymbol{k}}^{P E S}$, with $W_{\boldsymbol{k}}^{P E S}$ the integrated PES weight. Low weight in the shadow band allows on one hand to maintain the next-nearest neighbor dispersion of width $J$, which seems to be optimal for the exchange mediated propagation of a single hole [1], while at the same time staying close to the free-electron $W_{\boldsymbol{k}}^{P E S}$ (see Figure 3a) so as to optimize the 'ordinary' kinetic energy. We turn to the IPES spectrum, which consists of several components: the hole pockets right at $E_{F}$ near $(\pi / 2, \pi / 2)$ (or $(\pi, 0)$ for two holes in a small clusters [2]) and the various 'shake-off bands' at higher energy and predominantly in the outer part of the Brillouin zone. When viewed with coarse energy and/or $\boldsymbol{k}$ resolution, the resulting distribution of spectral weight may be rather similar to a 'renormalized free electron band' [4]; let us note that this is largely necessitated by elementary rules [8]. Key features which would allow for a distinction are a) the low intensity 'shadow bands' in the outer parts of the Brillouin zone, b) a 'disconnected' IPES spectrum consisting of low energy hole pockets near $(\pi / 2, \pi / 2)$ and higher energy magnon bands near $(\pi, \pi)$, and c) a Fermi surface with a volume proportional to the hole concentration $\delta$.

To address these issues, we proceed to a comparison with experiments on cuprate superconductors. PES experiments on the insulating antiferromagnet $\mathrm{Sr}_{2} \mathrm{CuO}_{2} \mathrm{Cl}_{2}$ [9] have given evidence for a dramatic shadow band effect in this undoped antiferromagnet. Assuming that the weight of the shadow bands even decreases in the doped case (as is suggested by cluster studies [2,6]) would readily explain the non-observation of the shadow bands in early ARPES experiments. A recent Fermi surface mapping of the metallic compound $\mathrm{Bi}_{2} \mathrm{Sr}_{2} \mathrm{CaCu}_{2} \mathrm{O}_{8+x}$ with an extremely high density of $\boldsymbol{k}$-points by Aebi et al. 10] has indeed shown indications for the shadow bands also in the doped case, a result which has been corroborated by conventional ARPES in the meantime [11].

We turn to IPES, where experimental data are more sparse than in the PES case. IPES experiments which revealed several dispersive bands were reported by Bernhoff et al. 12]. Figure 3b shows the positions of IPES peaks found by these authors in $\mathrm{Bi}_{2} \mathrm{Sr}_{2} \mathrm{CaCu}_{2} \mathrm{O}_{8}$, as well as an assignment of bands compatible with our scenario. Grouping the higher lying peaks as indicated in the figure, one can obtain almost quantitative agreement with the LDA band structure [13], provided the two $\mathrm{CuO}_{2}$ plane derived bands are omitted from the latter. We are then left with only two 'disconnected' low energy features: the extreme low energy intensity near $(\pi / 2, \pi / 2)$, which we associate with the hole pocket plus some 'isolated' spectral weight near $(\pi, \pi)$. The latter feature is most clearly visible at the largest possible distance from $\boldsymbol{k}_{F}$ and seems to disappear when approaching $\boldsymbol{k}_{F}$. This is in rather clear contrast to the behaviour expected for a 'renormalized free-electron band', which on the contrary should be most clearly visible near $\boldsymbol{k}_{F}$. On the other hand, this is precisely the behaviour expected for the magnon bands in the $t-J$ model, whose large intensity near $(\pi, \pi)$ is favourable to lower the kinetic energy. We turn to the volume of the Fermi surface. Here the most direct probe the deHaas-vanAlphen effect, which is insensitive to the quasiparticle weight $Z$ and hence may detect the 'shadow' sheets of the Fermi surface. Modelling the holes as spin 1/2 particles distributed evenly over 4 symmetric pockets of equal size, the fraction of the Brillouin zone covered by a single pocket is $\delta / 8$. With a lattice constant of $3.85 \AA$ (as would be appropriate for most cuprate superconductors) $\delta=0.18$ gives a de Haasvan Alphen cross-section of $0.63 k T$, which is roughly half-way in between two orbits of $(0.53 \pm 0.02) k T$ and $(0.78 \pm 0.02) k T$ actually observed in $\mathrm{YBa}_{2} \mathrm{Cu}_{3} \mathrm{O}_{6.97}$ [14. This suggests to assume that the two ' $t-J$ bands' derived from the two $\mathrm{CuO}_{2}$ planes/unit cell form bonding and antibonding combinations so that there is a disproportionation of holes between these two bands. With $\delta_{1}=0.15$, $\delta_{2}=0.22$ (i.e. an average $\delta$ of 0.185 ) one obtains orbits of $0.52 k T$ and $0.76 k T$, consistent with experiment [14]. For completeness we note that the experimental doping dependence of the low temperature Hall constant, which is another $Z$-independent quantity directly related to the carrier concentration, can also be reproduced well within the hole-pocket picture 15, 16 .

. In summary, we have presented a cluster diagonalization study of the inverse photoemission spectrum for the low doping regime of the $t-J$ model. The results first of all show a remarkable continuity with doping which, together with similar results for the photoemission spectra [2] and dynamical correlation functions [5] suggests that the single hole is the key problem for understanding the moderately doped region. The emerging picture of the single particle spectral function is that of a spectral weight distribution which in the neighborhood of the Fermi surface roughly resembles that of free electrons, combined with a hole-pocket Fermi surface with a volume proportional to the number of doped holes. Various experimental data which suggest that this in cuprate superconductors are consistent with this scenario.

Financial support of R. E. through the European Community is most gratefully acknowledged. Computations were carried out at the Institute for Molecular Science, Okazaki.

Figure 1: IPES spectrum $A_{+}(\boldsymbol{k}, \omega) / n_{h}$ for different $\boldsymbol{k}$ and $J / t$ for $n_{h}=1$ (a) and $n_{h}=2(\mathrm{~b})$. $\delta$-functions are replaced by Lorentzians of width $0.1 J$.

Figure 2: (a) IPES spectrum $A_{+}(\boldsymbol{k}, \omega)$ (full line), "interference spectra" $0.5 \cdot B_{1}(\boldsymbol{k}, \omega)$ (dashed line) and $B_{2}(\boldsymbol{k}, \omega)$ (dotted line), for $n=1$. The ratio $J / t=0.4, \delta$ functions are replaced by Lorentzians of width $0.01 J$. (b) Same as (a) for $n=2$. 
Figure 3: (a) Schematic spectral function of the $t-J$ model near the Fermi energy. The full line denotes the PES intensity from the quasiparticle band, the dashed line the IPES spectrum from the 'hole pocket', the short-dashed line the 'shake-off bands'. (b) Comparison of experimental and theoretical band structure of $\mathrm{Bi}_{2} \mathrm{Sr}_{2} \mathrm{CaCu}_{2} \mathrm{O}_{8}$. The Fermi level is the zero of energy, the symbols denote prominent peaks in the IPES spectrum 12 (above $E_{F}$ ) and in the PES spectrum [11] (below $\left.E_{F}\right)$. Dashed lines show the unoccupied part of the LDA bandstructure [13], with the $\mathrm{CuO}_{2}$-plane derived bands removed, and rescaled and shifted according to $E=0.77 \cdot\left(E_{L D A}-E_{F}\right)-0.32 \mathrm{eV}$. The full line indicates the ' $t-J$ band'. A group of PES peaks originating from the superlattice structure of $\mathrm{Bi}_{2} \mathrm{Sr}_{2} \mathrm{CaCu}_{2} \mathrm{O}_{8}$ 11 is omitted.
[1] For a recent review see E. Dagotto, Rev. Mod. Phys. 66, 763 (1994).

[2] R. Eder, Y. Ohta, and T. Shimozato, Phys. Rev. B 50, 3350 (1994).

[3] R. Eder and Y. Ohta, Phys. Rev. B 51, 6041 (1995); S. Haas, ibid. 11748; D. Duffy and A. Moreo, ibid. 11882.

[4] W. Stephan and P. Horsch, Phys. Rev. Lett. 66, 2258 (1990); E. Dagotto at al., Phys. Rev. B 46, 3183 (1992).

[5] R. Eder, Y. Ohta, and S. Maekawa, Phys. Rev. Lett. 74, 5124 (1995).

[6] A. Moreo et al., Phys. Rev. B 51, 12045 (1995).

[7] A. Kampf and J. R. Schrieffer, Phys. Rev. B 41, 6399 (1990).

[8] R. Eder and Y. Ohta, Phys. Rev. Lett. 72, 2816 (1994).

[9] B. O. Wells et al., Phys. Rev. Lett. 74, 964 (1995).

[10] P. Aebi et al., Phys. Rev. Lett. 72, 2757 (1993).

[11] H. Ding et al., SISSA preprint cond-mat/9507020.

[12] H. J. Bernhoff et al., Europhys. Lett. 13, 537 (1990).

[13] S. Massidda et al., Physica C 152, 251 (1988).

[14] C. M. Fowler et al., Phys. Rev. Lett. 68, 534 (1992).

[15] S. A. Trugman, Phys. Rev. Lett. 65, 500 (1990).

[16] E. Dagotto et al., Phys. Rev. Lett. 73, 728 (1994). 
Figure 1

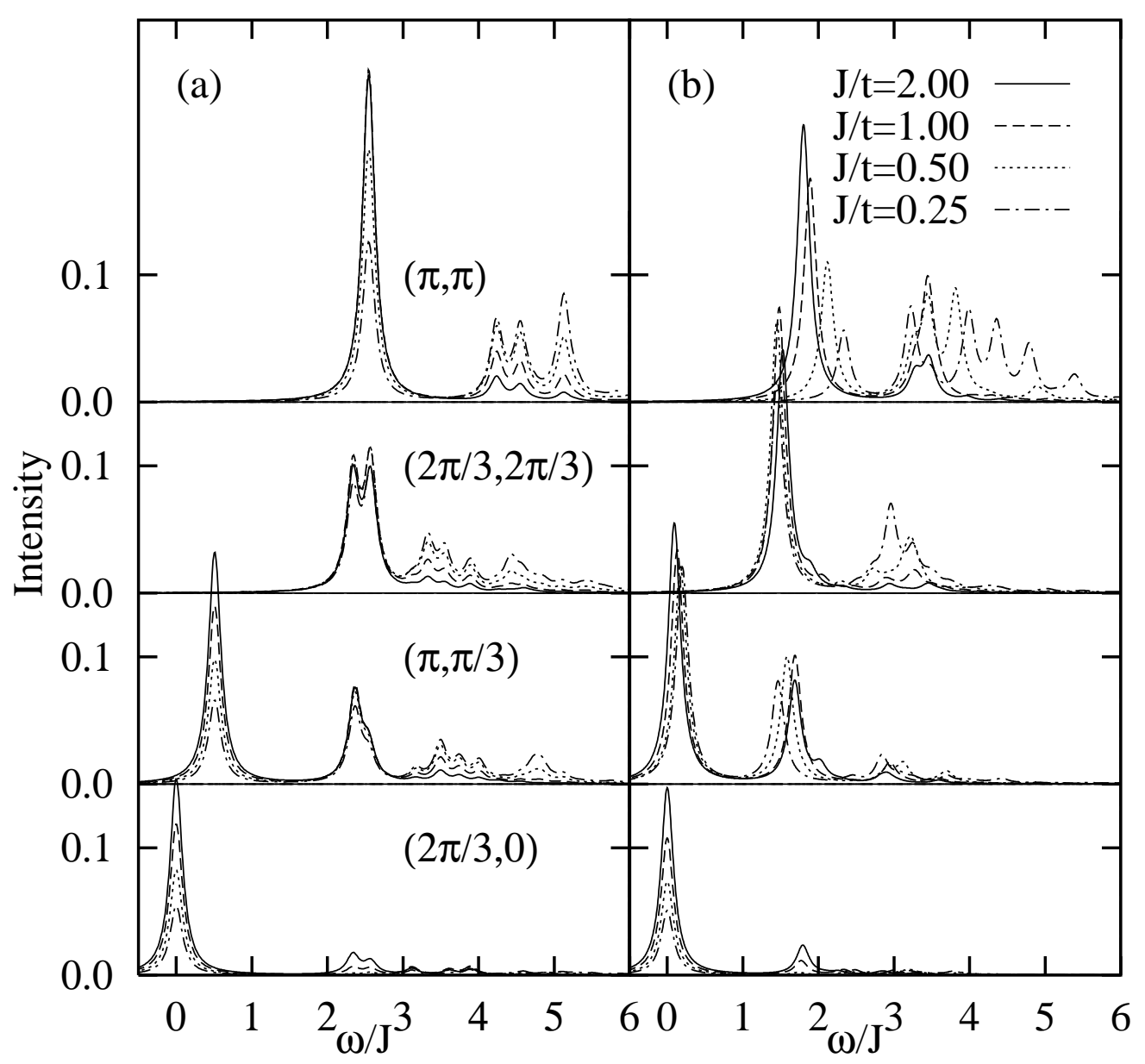


Figure 2

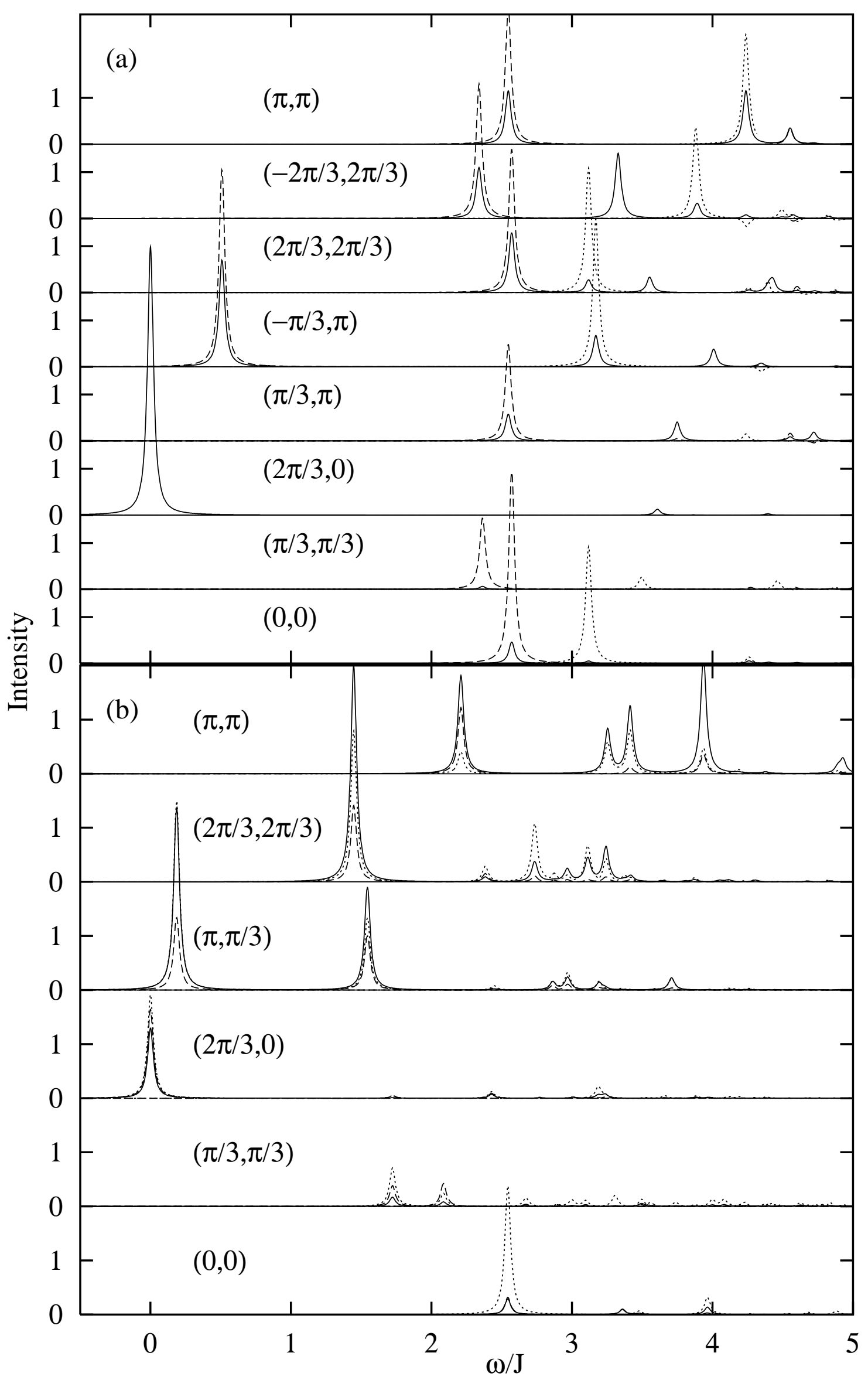


Figure 3
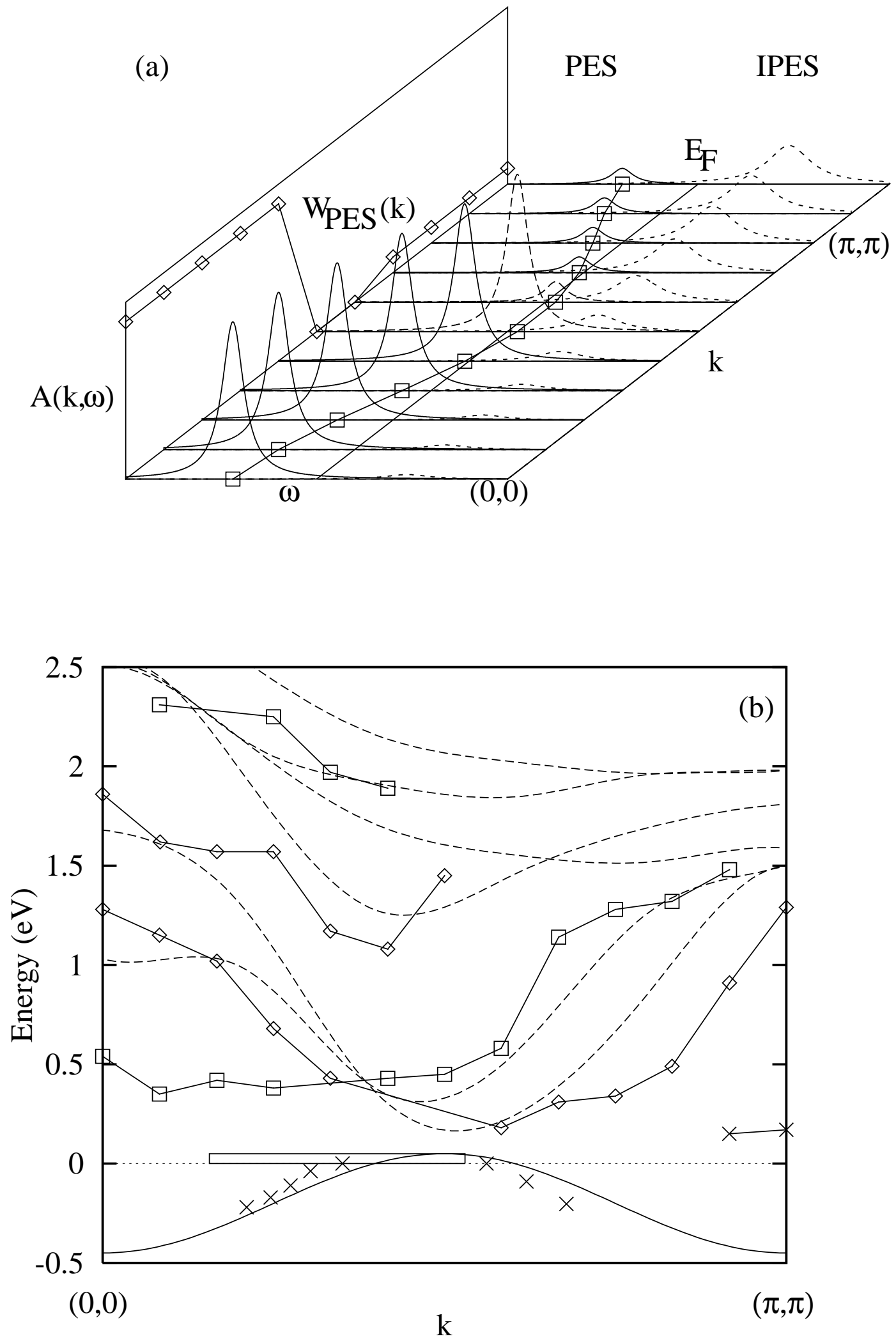